\title{
AVALIAÇÃO DA AMPLITUDE ARTICULAR DO JOELHO: CORRELAÇÃO ENTRE AS MEDIDAS REALIZADAS COM O GONIÔMETRO UNIVERSAL E NO DINAMÔMETRO ISOCINÉTICO
}

\author{
Batista LH ${ }^{1}$, Camargo PR ${ }^{1}$, Aiello GV ${ }^{1}$, Oishi $\mathrm{J}^{2}$, Salvini TF ${ }^{1}$ \\ ${ }^{1}$ Unidade de Plasticidade Muscular, Laboratório de Neurociências, Departamento de Fisioterapia, Universidade Federal \\ de São Carlos - UFSCar, São Carlos, SP \\ ${ }^{2}$ Departamento de Estatística, UFSCar, São Carlos, SP \\ Correspondência para: Tania F. Salvini,Universidade Federal de São Carlos, Departamento de Fisioterapia, Rodovia \\ Washington Luís, km 235, CEP 13565-905, São Carlos, SP, e-mail: tania@power.ufscar.br
}

Recebido: 15/02/2005 - Aceito: 30/11/2005

\begin{abstract}
RESUMO
Contextualização: O instrumento mais utilizado pelos terapeutas para mensuração da amplitude de movimento (ADM) articular é o goniômetro universal. No entanto, há carência de estudos que analisem a confiabilidade das medidas da ADM do joelho realizadas no dinamômetro isocinético. Objetivo: O objetivo deste estudo foi analisar a correlação entre as medidas de ADM na articulação do joelho, realizadas com o goniômetro universal e no dinamômetro isocinético. Método: Foram avaliados 38 voluntários saudáveis (27 mulheres, 11 homens), com idade de $36 \pm 11$ anos, com limitação mínima de $20^{\circ}$ na ADM de extensão do joelho. No membro dominante de cada sujeito foram realizadas três mensurações da ADM do joelho com o goniômetro e três mensurações no dinamômetro. Resultados: Os resultados deste estudo mostraram que há alto grau de correlação entre as medidas da ADM do joelho obtidas com o goniômetro universal e no dinamômetro isocinético (Coeficiente de Correlação de Pearson = 0,90). Conclusão: Com os procedimentos realizados, tanto o goniômetro universal como o dinamômetro isocinético podem ser utilizados para avaliação da ADM do joelho, pois ambos apresentam mensurações confiáveis.
\end{abstract}

Palavras-chave: goniômetro, dinamômetro isocinético, joelho, amplitude de movimento.

\section{ABSTRACT \\ Knee Joint Range-of-Motion Evaluation: Correlation Between Measurements Achieved Using a Universal Goniometer and an Isokinetic Dynamometer}

Background: The instrument most often used by therapists for measuring joint range of motion (ROM) is the universal goniometer. However, there is a lack of studies analyzing the reliability of knee joint ROM measured by the isokinetic dynamometer. Objective: The purpose of this study was to analyze the correlation between the knee joint ROM measurements made using a universal goniometer and an isokinetic dynamometer. Method: 38 healthy volunteers (27 women, 11 men) aged $36 \pm 11$ years were evaluated. All of them had a minimum knee extension ROM limitation of $20^{\circ}$. Three knee ROM measurements were made using the universal goniometer and another three using the isokinetic dynamometer, on each subject's dominant limb. Results: The results showed a high degree of correlation between the knee ROM measurements made using the two instruments (Pearson correlation coefficient $=0.90)$. Conclusion: From the procedures performed, both the universal goniometer and the isokinetic dynamometer can be used to evaluate knee ROM, since they both present reliable measurements.

Key words: goniometer, isokinetic dynamometer, knee, ROM. 


\section{INTRODUÇÃO}

A medida da amplitude de movimento articular (ADM) é um componente importante na avaliação física, pois identifica as limitações articulares, bem como permite aos profissionais acompanharem de modo quantitativo a eficácia das intervenções terapêuticas durante a reabilitação. O instrumento mais utilizado pelos terapeutas para medir a ADM é o goniômetro universal ${ }^{1}$. No entanto, há também outros instrumentos capazes de mensurar a ADM, como o dinamômetro isocinético ${ }^{2}$. Nas últimas décadas houve um grande aumento no uso deste instrumento em várias áreas, dentre elas a fisioterapia, que tem se beneficiado de forma particular e significativa desta tecnologia ${ }^{3}$. Para que o goniômetro universal e o dinamômetro isocinético possam ser correlacionados e utilizados na mensuração da ADM, é necessário que ambos forneçam medidas confiáveis.

Segundo Dvir ${ }^{3}$, a confiabilidade de uma medida é a consistência entre as medidas sucessivas da mesma variável, no mesmo sujeito e nas mesmas condições. O autor cita, ainda, que há três fontes de erro que podem tornar uma avaliação não confiável: o instrumento de medida, a pessoa que ministra a avaliação e as diferentes características dos voluntários que estão sendo avaliados que é, sem dúvida, a fonte de erro mais difícil de controlar. Considerando-se as diferenças individuais, as medidas obtidas por meio de um equipamento podem variar, sendo assim, a confiabilidade destas medidas só será confirmada após a aplicação de testes estatísticos específicos ${ }^{3,4,5}$.

A goniometria, descrita na literatura desde 1914, é amplamente usada, tanto na prática clínica quanto em pesquisas científicas, com a finalidade de medir a ADM de diversas articulações. Vários experimentos examinaram o grau de confiabilidade das medidas goniométricas utilizando diferentes procedimentos de medida e demonstraram que a ADM do joelho, medida com goniômetro universal, obteve um nível de confiabilidade de bom a excelente ${ }^{6-14}$. Outros trabalhos concluíram serem válidas as medidas realizadas com o goniômetro universal, após terem sido correlacionadas às medidas da ADM obtidas a partir de radiografia, considerada um padrão de medida bem estabelecido ${ }^{11,12,14}$. Sendo assim, o goniômetro universal é um instrumento com o qual se obtém medidas da ADM do joelho confiáveis e válidas. Entretanto, Ellis e Bruton ${ }^{15}$ concluíram que as medidas de $\mathrm{ADM}$ de articulações da mão realizadas com o goniômetro são mais confiáveis quando somente uma medida é envolvida que quando múltiplas medidas articulares são necessárias. Brosseau ${ }^{14}$ cita que as medidas goniométricas da ADM do joelho são mais confiáveis quando realizadas pelo mesmo avaliador. Aalto et al. ${ }^{8}$, em um estudo recente, verificaram que as medidas realizadas na articulação do joelho com o goniômetro obtiveram baixa confiabilidade, mas em contrapartida comentam que o posicionamento utilizado para o teste pode ter contribuído para este resultado. Finalmente, o goniômetro é um instrumento de medida articular mais confiável ${ }^{16}$ e válido ${ }^{14}$ que a estimativa visual.

A ADM de vários segmentos corporais também pode ser mensurada no dinamômetro isocinético, um equipamento computadorizado bastante utilizado para avaliação funcional do sistema musculoesquelético. No entanto, há carência de estudos na literatura que analisem a confiabilidade das medidas da ADM realizadas no dinamômetro isocinético.

Considerando que a articulação do joelho é uma das mais lesadas no ser humano, diferentes procedimentos para a mensuração de sua ADM são utilizados. Tais medidas podem ser realizadas pelo terapeuta utilizando o goniômetro universal manual, ou ainda, por meio de instrumentos computadorizados, como o dinamômetro isocinético. Assim, seria importante verificar se as medidas fornecidas por estes dois meios de mensuração apresentam correlação entre si.

O objetivo deste estudo foi analisar o grau de correlação entre as medidas de ADM de extensão do joelho, realizadas pelo terapeuta com o goniômetro universal, às obtidas com o dinamômetro isocinético.

\section{METODOLOGIA}

\section{Voluntários}

Participaram deste estudo 38 voluntários saudáveis, de ambos os sexos (27 mulheres e 11 homens) com idade de $36 \pm 11$ anos. Como critério de inclusão, os voluntários deveriam apresentar limitação de $20^{\circ}$ na $\mathrm{ADM}$ de extensão do joelho ${ }^{17}$ do membro dominante, mensurado com o goniômetro universal, com o sujeito posicionado em decúbito dorsal e as articulações do quadril e joelho fletidas a $90^{\circ}{ }^{13}$. Todos os voluntários foram informados sobre os objetivos e procedimentos do estudo e assinaram um termo de consentimento livre e esclarecido conforme resolução 196/96 do Conselho Nacional de Saúde. O estudo foi aprovado pelo Comitê de Ética da Universidade para estudos em humanos.

\section{Instrumentação}

Para avaliar a ADM de extensão do joelho foram utilizados um goniômetro universal de material plástico (Carci) e um dinamômetro isocinético (Biodex Multi-joint System $3)$.

\section{Procedimentos}

Medida da ADM de extensão do joelho com o goniômetro universal

Com o objetivo de diminuir possíveis diferenças nas avaliações pelo terapeuta, duas fisioterapeutas foram previamente treinadas e realizaram todas as avaliações da ADM do joelho com o goniômetro universal e no dinamômetro isocinético deste estudo.

Inicialmente, com os indivíduos em posição dorsal foi realizado um teste para verificar a presença de encurtamento 
dos músculos flexores do quadril, como descrito por Kendall ${ }^{17}$, que pode causar compensações, como a anteversão pélvica, e alterar a mensuração da ADM do joelho. Assim, nos indivíduos que apresentaram encurtamento dos flexores do quadril, a articulação do joelho do membro não dominante foi fletida, com auxílio de almofadas, mantendo a pelve em posição neutra para evitar compensações (Figura 1).

Para a avaliação inicial, o quadril e o joelho do membro dominante foram fletidos a $90^{\circ}$ e o pé mantido relaxado ${ }^{13}$. A partir desta posição, o joelho foi passiva e lentamente estendido pelo avaliador 1 , enquanto o avaliador 2 certificavase de que não estavam ocorrendo compensações. O voluntário foi orientado a relaxar durante a avaliação, particularmente quando seu joelho estava sendo estendido, e relatar o momento em que sentiu o início da tensão nos músculos flexores do joelho, que foi considerado a posição final (Figura 1). Atingida esta posição, o avaliador 1 mensurou o grau de encurtamento dos flexores do joelho, ou seja, a extensão do joelho, com o goniômetro universal (Figura 1), conforme descrito por Norkin e White ${ }^{1}$. A extensão completa do joelho foi considerada $0^{\circ}{ }^{13}$ e utilizada como referência para o cálculo do grau de limitação da extensão articular do joelho.

A medida da ADM extensora do joelho de cada indivíduo foi avaliada três vezes, sendo utilizada a média aritmética das três mensurações.

Medida da ADM de extensão do joelho no dinamômetro isocinético

Para manter o quadril dos voluntários fletido a aproximadamente $90^{\circ}$, um acessório acolchoado foi acoplado

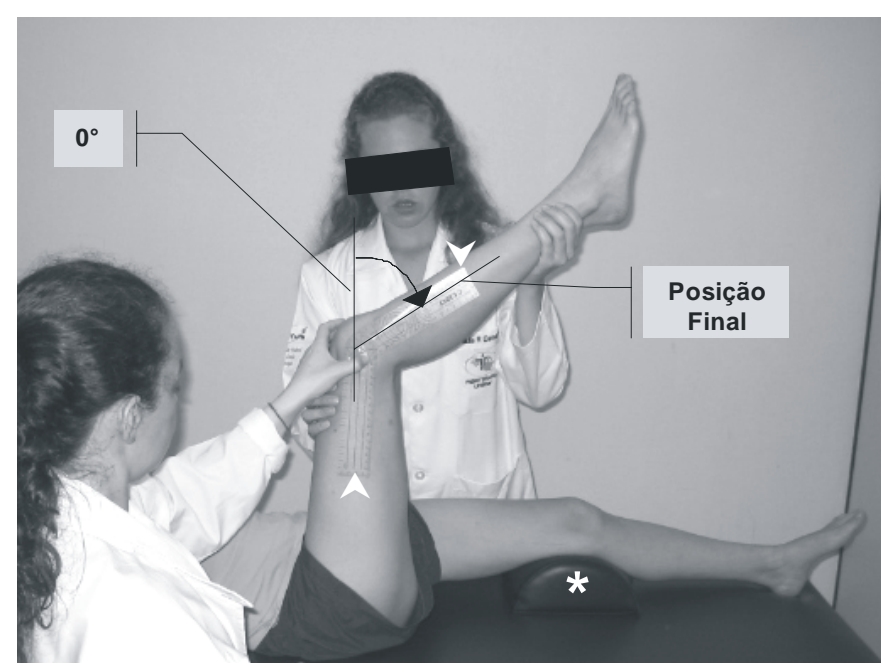

Figura 1. Avaliação da ADM de extensão do joelho com o goniômetro universal (indicação com cabeças de seta branca), que indica o grau de encurtamento dos músculos flexores desta articulação. A mensuração da ADM foi realizada quando o indivíduo relatou o início de tensão nos músculos flexores do joelho. Almofada $\left(^{*}\right)$ utilizada em indivíduos com encurtamento nos flexores do quadril do membro contralateral, para manter a pelve em posição neutra. ao encosto da cadeira do dinamômetro (Figura 2). Este procedimento garantiu que o mesmo posicionamento neutro da pelve, utilizado durante a avaliação da ADM do joelho com o goniômetro, fosse também mantido durante as mensurações realizadas no dinamômetro.

O voluntário foi estabilizado na cadeira do dinamômetro por meio de cintos de contenção sobre o tronco, quadril e na coxa do membro dominante avaliado (Figura 2). A seguir, o eixo mecânico de rotação do dinamômetro foi alinhado com o epicôndilo lateral do fêmur e a perna foi fixada ao braço de resistência do dinamômetro, mantendo livre a articulação do tornozelo.

Previamente à realização da avaliação, os voluntários receberam um dispositivo manual ligado ao dinamômetro (Figura 2), por meio do qual o voluntário poderia iniciar ou parar a avaliação passiva que estava sendo realizada. Em seguida, foram orientados a fechar os olhos e se manterem relaxados. Após isso, deveriam acionar o dinamômetro, por meio do dispositivo, para que o braço de resistência do equipamento começasse a estender o joelho, passivamente,

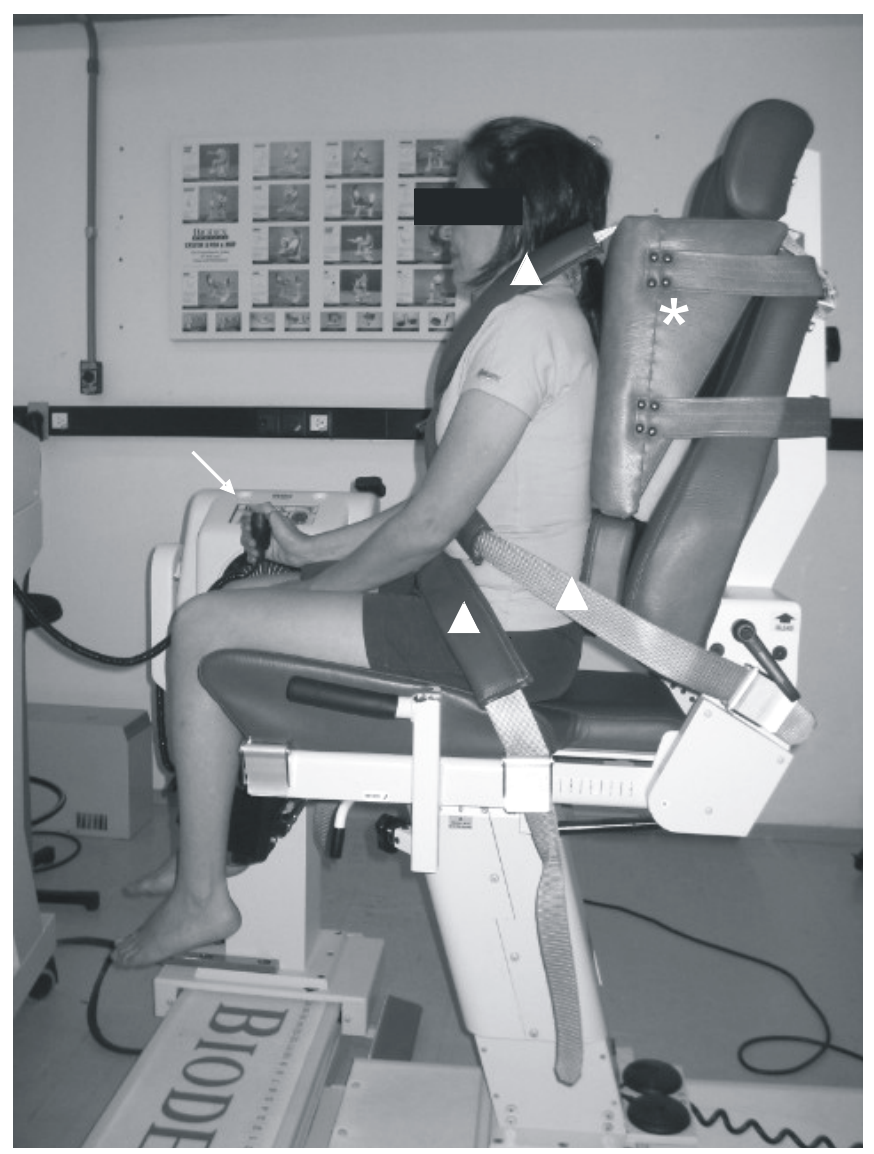

Figura 2. Posicionamento do voluntário na cadeira do dinamômetro: cintos de estabilização $(\Delta)$, dispositivo (seta) utilizado para iniciar ou finalizar a avaliação passiva, e acessório (*) para manter a articulação do quadril a aproximadamente $90^{\circ}$ de flexão. 
a $2 \%$ de velocidade. Nesta fase, o voluntário deveria manterse totalmente passivo ao movimento. Também foram orientados a parar o braço de resistência do dinamômetro, por meio do dispositivo, assim que sentissem iniciar a tensão de alongamento nos músculos flexores do joelho, para mensuração da ADM extensora dessa articulação. Foram realizadas três mensurações consecutivas desse movimento e sua média aritmética foi utilizada para a análise estatística.

\section{Análise estatística}

O Coeficiente de Variação (CV) foi usado inicialmente para estimar a porcentagem de variação entre as medidas realizadas na primeira, segunda e terceira avaliação da ADM extensora do joelho obtidas com os dois instrumentos de avaliação utilizados.

A confiabilidade e a correlação das medidas da ADM do joelho, realizadas com o goniômetro e no dinamômetro, foram analisadas estatisticamente por meio dos testes: Coeficiente de Variação (CV) e Coeficiente de Correlação de Pearson (CCP).

O CV foi aplicado para obter a variação intervoluntários e intravoluntários. Para cada instrumento de avaliação, o CV intervoluntários foi considerado como referência para o CV intravoluntários, de acordo com Rothstein ${ }^{9}$ e Norkin \& White ${ }^{1}$. Considerou-se que CCP com valores de 0,90 a 0,99 corresponde à alta correlação, de 0,80 a 0,89 a boa correlação, de 0,70 a 0,79 a pequena correlação e valores $\leq 0,69$ uma baixa correlação ${ }^{18}$.

\section{RESULTADOS}

Com base nos resultados apresentados na Tabela 1, pode-se observar que o CV da primeira mensuração é maior que das duas últimas avaliações em ambos os instrumentos de medida, sendo ligeiramente maior no dinamômetro, embora essa diferença não seja significativa. Verifica-se também que, de modo geral, os valores do CV referentes às três avaliações realizadas no dinamômetro isocinético variaram mais que as realizadas com o goniômetro universal.

Os resultados mostram que o CV intravoluntários obtido foi menor que o CV intervoluntários, nos dois instrumentos de avaliação da ADM articular (Tabela 2).

O Coeficiente de Correlação de Pearson indicou um alto grau de correlação $(0,90 ; p<0,05)$ entre as medidas obtidas com o goniômetro e no dinamômetro (Figura 3).

\section{DISCUSSÃO}

Considerando as condições experimentais utilizadas, os resultados do presente estudo revelam que as medidas da ADM extensora do joelho realizadas com o goniômetro universal têm alta correlação às obtidas no dinamômetro isocinético. Estudos têm utilizado o goniômetro universal para testar a confiabilidade das medidas da ADM, de várias articulações, utilizando diferentes instrumentos de medida ${ }^{14,19}$ - 21, mas não foram encontrados na literatura trabalhos que utilizassem o goniômetro universal para testar a correlação

Tabela 1. Resultados das mensurações da ADM de extensão do joelho realizados com o goniômetro universal e no dinamômetro isocinético, em 38 indivíduos.

\begin{tabular}{|c|c|c|c|c|c|c|}
\hline \multicolumn{4}{|c|}{ GONIÔMETRO UNIVERSAL } & \multicolumn{3}{|c|}{ DINAMÔMETRO ISOCINÉTICO } \\
\hline Mensurações & $\mathbf{1}^{\mathrm{a}}$ & $2^{a}$ & $3^{\mathrm{a}}$ & $\mathbf{1}^{\mathrm{a}}$ & $2^{\mathrm{a}}$ & $3^{a}$ \\
\hline $\mathbf{X} \pm \mathbf{D P}$ & $44,5 \pm 12,1^{\circ}$ & $45,0 \pm 11,9^{\circ}$ & $44,9 \pm 11,8^{\circ}$ & $46,3 \pm 15,4^{\circ}$ & $46,6 \pm 13,6^{\circ}$ & $45,8 \pm 14,0^{\circ}$ \\
\hline CV & $27,2 \%$ & $26,4 \%$ & $26,4 \%$ & $33,1 \%$ & $29,2 \%$ & $30,6 \%$ \\
\hline
\end{tabular}

X = média; DP = desvio-padrão; CV = coeficiente de variação.

Tabela 2. Comparação da avaliação intervoluntários e intravoluntários da ADM de extensão do joelho, obtida com goniômetro universal e dinamômetro isocinético, em 38 indivíduos.

\begin{tabular}{cccccc}
\hline & $\mathbf{X}$ & $\mathbf{D P}^{\mathbf{4}}$ & $\mathbf{D P}$ & $\mathbf{C V}^{\mathbf{4}}$ & $\mathbf{C V}^{\mathbf{*}}$ \\
\hline Goniômetro & $44,8^{\circ}$ & $10,7^{\circ}$ & $1,8^{\circ}$ & $23,9 \%$ & $4,0 \%$ \\
Dinamômetro & $46,2^{\circ}$ & $12,6^{\circ}$ & $3,7^{\circ}$ & $27,2 \%$ & $8,0 \%$ \\
\hline
\end{tabular}

$\mathrm{X}$ = média; DP = desvio-padrão; CV = coeficiente de variação; ^ intervoluntários,

- intravoluntários 


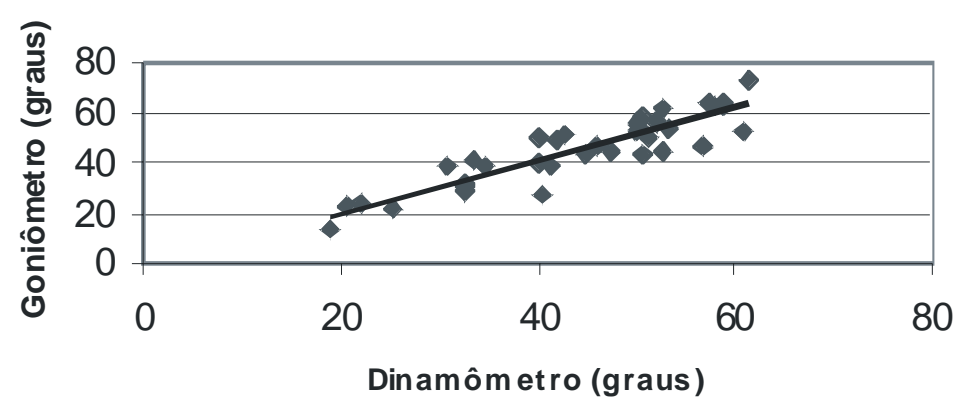

Figura 3. Valores do Coeficiente de Correlação de Pearson $(0,90)$ entre as medidas da ADM de extensão do joelho realizadas com o goniômetro universal e no dinamômetro isocinético, em 38 indivíduos.

das medidas de ADM do joelho realizadas no dinamômetro isocinético. Portanto, segundo nosso conhecimento, o resultado encontrado neste estudo é inédito na literatura.

Verificou-se que o CV intravoluntários das medidas realizadas tanto com o goniômetro quanto no dinamômetro, foram menores que o CV intervoluntários. Segundo Rothstein ${ }^{9}$ e Norkin e White ${ }^{1}$, estes resultados indicam que as medidas da ADM do joelho realizadas em ambos os instrumentos são confiáveis. Portanto, há uma alta confiabilidade das medidas realizadas tanto manualmente pelo terapeuta, com o goniômetro universal, quanto às realizadas no dinamômetro isocinético (Tabela 2).

No entanto, alguns aspectos observados necessitam ser considerados. Os resultados indicaram que há diferença entre os CV das medidas realizadas com o goniômetro e no dinamômetro (Tabela 2). O CV, intervoluntários e intravoluntários, das medidas obtidas no dinamômetro foram maiores do que as realizadas com o goniômetro. Segundo Norkin e White ${ }^{1}$, vários fatores podem alterar o grau de confiabilidade de uma medida. Tais fatores foram considerados neste estudo para que os procedimentos realizados durante a mensuração da ADM extensora do joelho no dinamômetro fosse o mais próximo possível dos procedimentos de mensuração com o goniômetro, são eles: ambos os métodos de medida foram realizados pelos mesmos avaliadores, as duas formas de avaliação mantiveram a articulação do quadril fletido a aproximadamente $90^{\circ}$, a ADM extensora do joelho foi medida de forma passiva nos dois métodos, e foram mantidos os mesmos números de repetições. Embora todos esses cuidados tenham sido tomados, com o objetivo de minimizar possíveis diferenças nos procedimentos experimentais entre as mensurações nos dois instrumentos de avaliação, observou-se que as mensurações no dinamômetro isocinético variaram mais, quando comparadas às do goniômetro universal. Esta variação pode ter ocorrido, segundo relato dos voluntários, pela maior dificuldade em sentir o início da tensão nos músculos flexores do joelho durante a mensuração da ADM realizada no dinamômetro. Tal dificuldade, provavelmente, foi a causa da maior variação entre as medidas da $\mathrm{ADM}$ obtidas neste equipamento, quando comparadas às medidas com o goniômetro universal. Esse resultado indica a necessidade de um número maior de repetições quando a avaliação da ADM articular for realizada no dinamômetro isocinético. Esta sugestão é reforçada pelos resultados da Tabela 1 que mostra o CV das três avaliações referentes a cada instrumento de medida. Pode-se observar que a primeira avaliação variou mais que as subseqüentes, independentemente do instrumento utilizado para a mensuração, sendo essa variabilidade maior no dinamômetro isocinético. Este resultado demonstra a necessidade de realizar familiarização prévia dos voluntários com os instrumentos de medida, particularmente com o dinamômetro.

É importante ressaltar ainda que durante a execução do presente estudo, foi verificado que $20 \%$ dos voluntários apresentaram alterações na percepção da tensão dos músculos flexores do joelho, com uma variação média de $22^{\circ}$ entre as medidas realizadas com o goniômetro e no dinamômetro, enquanto que $80 \%$ dos sujeitos variaram $4^{\circ}$. Esses voluntários foram reavaliados, utilizando-se a mesma posição de mensuração, ou seja, tanto as medidas realizadas com o goniômetro, quanto no dinamômetro foram obtidas na posição sentada. A variação entre as medidas da ADM de extensão do joelho passou de $22^{\circ}$ para $6^{\circ}$. Diante deste resultado, pode-se dizer que a grande dificuldade de percepção apresentada por $20 \%$ dos voluntários está relacionada à posição de mensuração. Sendo assim, deve-se considerar que a posição altera o grau de percepção de alguns voluntários e que esta dificuldade pode alterar a análise final das medidas obtidas por estes instrumentos.

Com a constatação da confiabilidade das medidas realizadas com ambos os instrumentos, foi possível, então, correlacioná-las.O valor do Coeficiente de Correlação de Pearson (CCP) encontrado indicou um alto grau de correlação entre as mensurações obtidas nos dois instrumentos. Sendo assim, tanto o CV quanto CCP mostraram que há um alto grau de confiabilidade das medidas dos dois equipamentos e entre eles. Tais resultados não corroboram com os encontrados 
por Farber et al. ${ }^{21}$ os quais verificaram que as medidas do ângulo articular realizadas com um equipamento computadorizado demonstraram ser mais confiáveis que as obtidas com o goniômetro.

Como em outros trabalhos, alguns utilizando procedimentos diferentes de medida ${ }^{14,22}$ e outros o mesmo procedimento $^{13,23,24}$, o presente estudo também mostra que o goniômetro é um instrumento que pode ser usado para realizar as medidas de ADM de extensão do joelho. Além disso, os resultados apresentados aqui demonstraram que o dinamômetro isocinético também é adequado para avaliar a ADM de extensão do joelho.

\section{CONCLUSÃO}

Considerando os procedimentos utilizados neste estudo, tanto o goniômetro universal como o dinamômetro isocinético podem ser utilizados para avaliação da ADM do joelho, pois suas mensurações são confiáveis.

Agradecimentos: Esse projeto recebeu apoio financeiro da FAPESP e do CNPq. Batista LH e Camargo PR receberam bolsa de Mestrado da CAPES, Aiello GV foi bolsista de Iniciação Científica do PIBIC-CNPq.

\section{REFERÊNCIAS BIBLIOGRÁFICAS}

1. Norkin CC, White DJ. Medida da amplitude de movimento articular: um guia para goniometria. Philadelphia: FA Davis Co; 1995.

2. Davies GJ. A compendium of isokinetics in clinical usage and rehabilitation techniques. $4^{\mathrm{a}}$ ed. Onalaska: WIS \& S Publishers; 1992.

3. Dvir Z. Isocinético: Avaliações musculares, Interpretações e Aplicações clínicas. $1^{\text {a }}$ ed. São Paulo: Manole; 2002.

4. Gleeson NP, Mercer TH. The utility of isokinetic dynamometry in the assessment of human muscle function. Sports Med 1996; 21(1): 18-34.

5. Greenfield MLVH, Kuhn EJ, Woijtys EM. A statistics primer: validity and reliability. Am J Sports Med 1998; 3 (26): 483-85.

6. Russell TN, Bandy WD. Eccentric training and static stretching improve hamstring flexibility of high school males. J Athl Train 2004; 39(3): 254-58.

7. Winters MV, Blake CG, Trost JS, Marcello-Brinker TB, Lowe L, Garber MB, et al. Passive versos active stretching of hip flexor muscles in subjects with limited hip extension: a randomized clinical trial. Phys Ther 2004; 84(9): 800-7.

8. Aalto TJ, Airaksinem O, Harkonen TM, Arokoski JP. Effect of stretch on reproducibility of hip range of motion measurements. Arch Phys Med Rehabil 2005; 86: 549-57.
9. Rothstein JM, Miller PJ, Roettger RF. Goniometric reliability in a clinical setting: Elbow and knee measurement. Phys Ther 1983; 63: 1611-15.

10. Enwemeka CS. Radiographic verification of knee goniometry. Scand J Rehabil Med 1986; 18: 47-50.

11. Gogia PP, Braatz JH, Rose SJ, Norton B J. Reliability and validity of goniometric measurements at the knee. Phys Ther 1987; 67: 192-5.

12. Herrmann DB. Validity study of head and neck flexion-extension motion comparing measurements of a pendulum goniometer and roentgenograms. J Orthop Sports Phys Ther 1990; 11: 414-20.

13. Bandy WD, Irion JM, Briggler M. The effect of time and frequency of static stretching on flexibility of the hamstring muscles. Phys Ther 1997; 77: 1090-6.

14. Brosseau L, Balmer S, Tousignant M, O’Sullivan JP, Goudreault $\mathrm{C}$, Goudreault M, et al. Intra and intertester reliability and criterion validity of the parallelogram and universal goniometers for measuring maximum active knee and extension of patients with knee restrictions. Arch Phys Med Rehabil 2001; 82(3): 396-402.

15. Ellis B, Bruton A. A study to compare the reliability of composite finger flexion with goniometry for measurement of range of motion in the hand. Clin Rehabil 2002; 16(5): 562-70.

16. Watkins MA. Reliability of goniometric measurements and visual estimates of knee range of motion obtained in clinical setting. Phys Ther 1991; 71(2): 90-6.

17. Kendall FP, Mccrery EK, Provance PG. Músculos: provas e funções. $4^{\text {a }}$ ed. São Paulo: Manole; 1995. p. 34.

18. Blech TE. Measurement in physical education. 1974; $2^{\mathrm{a}}$ ed. Ronald Press, New York. Cited in Currier, D. P. Elements of research in physical therapy. $3^{\text {a }}$ ed. Baltimore: Williams \& Wilkins; 1990.

19. Russell TG, Jull GA, Wootton R. Can the internet be used as a medium to evaluate knee angle? Man Ther 2003; 8(4):242-46.

20. Sprigle S, Flinn N, Wootten M, Mccrry S. Development and testing of a pelvic goniometer designed to measure pelvic tilt and hip flexion. Clin Biomech 2003; 18: 462-5.

21. Farber DC, Deorio JK, Steel MW. Goniometric versus computerized angle measurement in assessing hallux valgus. Foot Ankle Int 2005; 26(3): 234-8.

22. Willy RW, Kyle BA, Moore SM, Chleboun GS. Effect of cessation and resumption of static hamstring muscle stretching on joint range of motion. J Orthop Sports Phys Ther 2001; 31(3): 138-44.

23. Feland JB, Myrer JW, Schulthies SS, Fellingham GW, Measom GW. The effect of duration of stretching of the hamstring muscle group for increasing range of motion in people aged 65 years or older. Phys Ther 2001; 81(5): 1111-7.

24. Feland JB, Myrer JW, Merrill RM. Acute changes in hamstring flexibility: PNF versus static stretch in senior athletes. Phys Ther Sports 2001; 2: 186-93. 